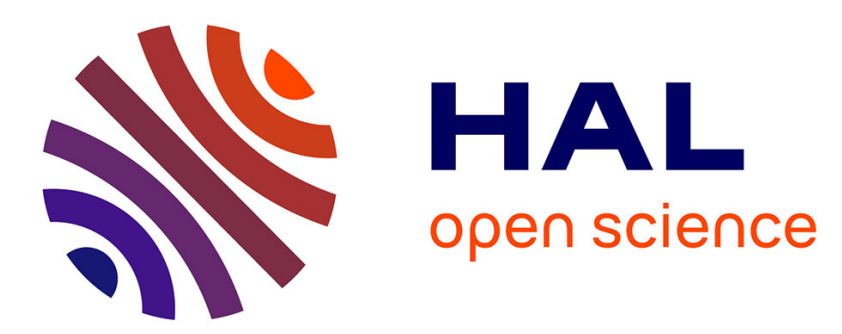

\title{
Improving the tuning of First-Order Autoregressive Model for the estimation of Amplify and Forward Relay channel
}

Soukayna Ghandour - Haidar, Laurent Ros, Jean-Marc Brossier

\section{- To cite this version:}

Soukayna Ghandour - Haidar, Laurent Ros, Jean-Marc Brossier. Improving the tuning of First-Order Autoregressive Model for the estimation of Amplify and Forward Relay channel. ICT 2012 - 19th International Conference on Telecommunications, Apr 2012, Jounieh, Lebanon. 6 p. hal-00701510

\author{
HAL Id: hal-00701510 \\ https://hal.science/hal-00701510
}

Submitted on 25 May 2012

HAL is a multi-disciplinary open access archive for the deposit and dissemination of scientific research documents, whether they are published or not. The documents may come from teaching and research institutions in France or abroad, or from public or private research centers.
L'archive ouverte pluridisciplinaire HAL, est destinée au dépôt et à la diffusion de documents scientifiques de niveau recherche, publiés ou non, émanant des établissements d'enseignement et de recherche français ou étrangers, des laboratoires publics ou privés. 


\title{
Improving the tuning of First-Order Autoregressive Model for the estimation of Amplify and Forward Relay channel
}

\author{
Soukayna GHANDOUR-HAIDAR, Laurent ROS, Jean-Marc BROSSIER
}

\begin{abstract}
This paper deals with the estimation of the Amplify-and-Forward channel. Considering two widely accepted Rayleigh links with Jakes' spectrum, a first-order autoregressive model $A R(1)$ is used to approximate the cascade of both links. A standard estimation algorithm is the Kalman filter. In this paper, we keep the choice of the AR(1)-Kalman filter, but we show that the method usually exploited in the literature to calculate the AR(1)-model parameter presents some disappointing results. We propose other values of the $A R(1)$-model parameter to improve the channel estimation, based on an off-line minimization of the asymptotic mean square error MSE for a given Doppler and signal to noise ratio. The simulation results show a considerable gain in terms of MSE of the well-tuned Kalman-based channel estimator, especially for the most common scenario of slowfading channel.
\end{abstract}

Index Terms - Amplify-and-Forward Relay, Channel estimation, Autoregressive model, Kalman Filter, Jakes' spectrum, Bessel function, Auto-correlation, Doppler Frequency.

\section{INTRODUCTION}

Recently, engineers switched to cooperative communications. The principal cause of this switching is the need to exploit spatial diversity at low cost or sometimes in small space. When we talk about cooperative communication, we have to mention the Multiple Input Multiple Output systems due to the relation between them. After the revolution of the MIMO systems, the engineers and researchers proposed a new way to benefit from the MIMO systems, without need of multiple antennas at the source and/or destination. The simplest way to do this is to use one relay, and to send one version of the signal from the unique antenna placed at the source, but to receive at the destination two versions of the signal, from the direct path and the via-relay path.

The cooperative communication is then summarized by a network composed of a source, a destination, and one or more relays. There are many types of relays, and the widely used types are the Amplify-and-Forward relay (AF) which amplifies the received signal and retransmits it, and the decode-andForward relay (DF) which decodes the received signal, then reencodes and retransmits it. We cite the Estimate-and-Forward relay (EF) [6] and Compress-and-Forward relay (CF) too [7]. The source-relay-destination and source-destination links should be independent, since their dependency expels the diversity benefits. Moreover, the source-relay and relaydestination links are supposed independent too [1], [2], [3], [4].

Each of the Source-Relay and Relay-Destination links is modeled by a Rayleigh fading channel model with Jakes' Doppler spectrum. This is the most accepted random model that represents temporal variations of the equivalent baseband channel complex gain (CG) [4], [5], [8], [11].

To estimate or equalize this channel, it is difficult to use directly this model, because it is not convenient for implementation and for computer simulation. In the literature, the authors of [9] demonstrate that the autoregressive model with order one $\mathrm{AR}(1)$ is convenient enough for such correlated fading channel, especially in case of narrowband Doppler fading processes. So usually, the AR(1) model, accompanied with the assumption of zero-mean additive white circular complex Gaussian noise (CAWGN), is used to approach the one-link channel and facilitate its manipulation [8], [10], [11], [13].

Up to now, the appropriateness of $\mathrm{AR}(1)$ model for the Amplify-and-Forward channel is not demonstrated. Although, the AR(1) model has been used in the literature to model the Amplify-and-Forward channel [2], [4]. This approximation has been used to track the true channel by a Kalman Filter (KF) in various wireless communication systems [2], [10], [11], [13], and sometimes by other particle filters [4], linear MMSE estimator [1], [3], or least square estimator [3]. In this work, we keep using the Kalman filter like in our previous work dealing with one-link channel in [11].

The use of an $\mathrm{AR}(1)$ model requires a good choice of the AR(1) coefficient. In many papers [2], [4], [13], the AR(1) coefficient calculation for a given normalized Doppler frequency is based on a same criterion, called Correlation Matching $(\mathrm{CM})$ in this paper. However, the problem of poorly tuned coefficient by Correlation Matching criterion has just recently been pointed out and analyzed in [10], [11] for the case of one-link flat fading Rayleigh channel with Jakes' spectrum. In this paper, we extend our previous one-link work in [11] to the case of cooperative communications and more specifically for Amplify-and-Forward channel. This channel is the product of two Rayleigh channels approached with an AR(1) model. The filter used for estimation is the Kalman Filter (KF) and the goal is to minimize the asymptotic variance of estimation error (MAV). So we find numerically the optimal AR(1) coefficient under the MAV criterion, without the CM constraint and compare the performances for both cases. The paper is organized as follows: we first write the mathematical model used to represent the channel in section II. We then find numerically the value of optimal coefficient for the criterion of 


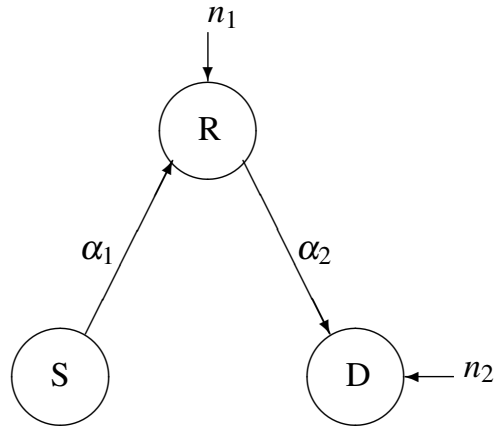

Figure 1. Relay-channel system model

minimizing the estimation error variance in section III.A. We also emphasize in section III.B and III.C the poor performance with the CM-based method, compared to the proposed method. Moreover, we study the behavior of the optimal MSE for a given Doppler and SNR scenario.

\section{Mathematical Model}

\section{A. Real Transmission}

We consider the estimation of the channel shown in figure 1 . It consists of a source $S$, a destination $D$, and a relay $R$ placed between them. We suppose that the direct link from source to destination is too poor, and we consider only the via-relay link. We divide our channel into two links, the Source-Relay link and the Relay-Destination link. Each of those links may be subject to be:

- A link where both extremities are mobile

- A link where one extremity is fixed while the other one is mobile

- A link where both of them are fixed

From the different scenarios that derivate, we focus on the mobile-fixed-mobile scenario. The source is moving at a speed of $v_{S}$, and the destination is moving at a speed of $v_{D}$. The relay is fixed. We can assume in this case that each link $(S-R$ and $R-D$ ) is a flat fading Rayleigh channel with Jakes' spectrum (Clarkes' model [12]), characterized by a complex gain CG $\alpha_{1}$ and $\alpha_{2}$ respectively, varying from one symbol to another, but we assume that the CG variation can be neglected during one symbol period. The variance of those gains are $\sigma_{\alpha_{1}}^{2}$ and $\sigma_{\alpha_{2}}^{2}$ respectively. For a sampling rate of $T$, the normalized Doppler frequency of the $S-R$ link is $f_{d 1} T$, where $f_{d 1}=\frac{v_{S}}{\lambda}$, and the normalized Doppler frequency of the $R-D$ link is $f_{d 2} T$, where $f_{d 2}=\frac{v_{D}}{\lambda} . \lambda$ is the wavelength of the analog emitted signal. A Jakes' Doppler spectrum is assumed for the $S-R$ link (the same will be for the $R-D$ link by replacing $f_{d 1}$ by $f_{d 2}$ and

$$
\left.\sigma_{\alpha_{1}}^{2} \text { by } \sigma_{\alpha_{2}}^{2}\right) \text { : }
$$

$$
\Gamma_{\alpha_{1}}(f)= \begin{cases}\frac{\sigma_{\alpha_{1}}^{2}}{\pi f_{d 1} \sqrt{1-\left(\frac{f}{f_{d 1}}\right)^{2}}} & \text { if }|f|<f_{d 1} \\ 0 & \text { if }|f|>f_{d 1}\end{cases}
$$

The autocorrelation coefficient for the $k^{\text {th }}$ tap of S-R link is given by:

$$
R_{\alpha_{1}}[k]=\sigma_{\alpha_{1}}^{2} J_{0}\left(2 \pi f_{d 1} T k\right)
$$

where $J_{0}$ is the zeroth-order Bessel function of the first kind. The same holds for the $R-D$ link.

The source sends the signal $s_{k}$. The relay receives the signal $r_{k}$, amplifies it with a factor of $A$ called relay gain, and retransmits the amplified signal $A . r_{k}$ to the destination. The destination receives the signal $y_{k}$ at the end. Since the gain of both links is constant during the period of the symbol, we observe a sampled and noised signal ( sampling at rate $T$ ).

$$
\begin{gathered}
r_{k}=s_{k} \alpha_{1 k}+n_{1 k} \\
y_{k}=A r_{k} \alpha_{2 k}+n_{2 k}
\end{gathered}
$$

where $n_{1 k}$ and $n_{2 k}, k=1,2, \ldots$ are Complex Additive White Gaussian Noises (CAWGN), with variances $\sigma_{n_{1}}{ }^{2}$ and ${\sigma_{n_{2}}}^{2}$ respectively.

The observation equation will be then

$$
y_{k}=s_{k} \alpha_{k}+n_{k}
$$

where $\alpha_{k}=A \alpha_{1 k} \alpha_{2 k}$ and $n_{k}=A \alpha_{2 k} n_{1 k}+n_{2 k}$, leading to

$$
\sigma_{\alpha}^{2}=A^{2}{\sigma_{\alpha_{1}}}^{2} \sigma_{\alpha_{2}}^{2}
$$

and

$$
\sigma_{n}^{2}=A^{2}{\sigma_{\alpha_{2}}}^{2}{\sigma_{n_{1}}}^{2}+{\sigma_{n_{2}}}^{2}
$$

The Gain $A$ is implicitly determined from the desired/required signal-to-noise ratio in the second link $\left(S N R_{2}\right)$ defined below, which is related to the energy of symbols emitted given $\sigma_{\alpha_{1}}^{2}$, $\sigma_{\alpha_{2}}^{2}, \sigma_{n_{1}}^{2}$ and $\sigma_{n_{2}}^{2}$. The signal $s_{k}$ is transmitted from the source with energy $E_{1}$. The energy $E_{2}$ of the signal $A r_{k}$ transmitted from relay is then

$$
E_{2}=A^{2}\left(E_{1} \sigma_{\alpha_{1}}{ }^{2}+\sigma_{n_{1}}{ }^{2}\right)
$$

The signal-to-noise ratio for the S-R link, R-D link, and all the channel are respectively given by :

$$
\begin{gathered}
\gamma_{1}=\frac{E_{1} \sigma_{\alpha_{1}}{ }^{2}}{\sigma_{n_{1}}{ }^{2}} \\
\gamma_{2}=\frac{E_{2} \sigma_{\alpha_{2}}{ }^{2}}{\sigma_{n_{2}}{ }^{2}} \\
\gamma=\frac{E_{1} \sigma_{\alpha}{ }^{2}}{\sigma_{n}{ }^{2}}=\frac{\gamma_{1} \gamma_{2}}{\gamma_{1}+\gamma_{2}+1}
\end{gathered}
$$

And the corresponding values in $(d B)$ are:

$$
\begin{aligned}
S N R_{1,2} & =10 \log _{10} \gamma_{1,2} \quad(d B) \\
S N R & =10 \log _{10} \gamma \quad(d B)
\end{aligned}
$$




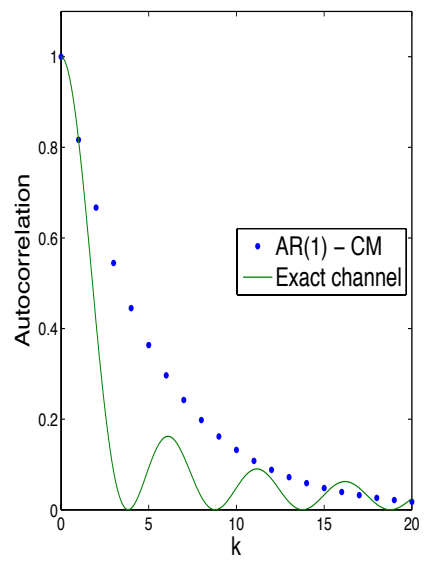

(a) $f_{d 1} T=f_{d 2} T=0.1$

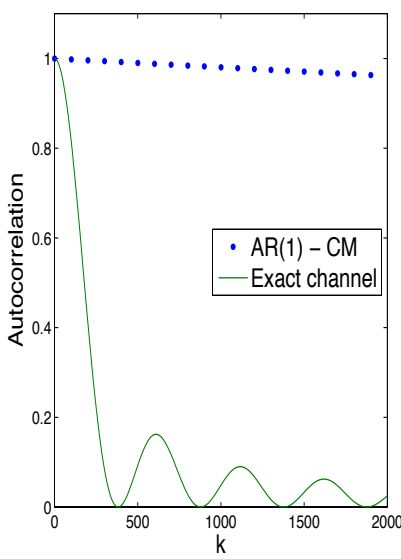

(b) $f_{d 1} T=f_{d 2} T=0.001$
Figure 2. Comparison of the auto-correlation functions of the true process $\alpha$ (continuous line) and the approached AR(1) process $\tilde{\alpha}$ for high and low Doppler frequencies (respectively left and right figures)

Both links are supposed independent. This leads to a total Doppler spectrum and total autocorrelation function [12], [4]:

$$
\begin{aligned}
& \Gamma_{\alpha}(f)=A \Gamma_{\alpha_{1}}(f) * \Gamma_{\alpha_{2}}(f) \\
R_{\alpha}[k]= & A^{2} R_{\alpha_{1}}[k] R_{\alpha_{2}}[k] \\
= & A^{2} \sigma_{\alpha_{1}}^{2} \sigma_{\alpha_{2}}^{2} J_{0}\left(2 \pi f_{d 1} T k\right) J_{0}\left(2 \pi f_{d 2} T k\right)
\end{aligned}
$$

We design by $*$ the convolution product.

\section{B. First-order autoregressive dynamical model}

In the literature, the time-varying $\mathrm{CG} \alpha_{k}$ is often approached by a first-order autoregressive $(\operatorname{AR}(1))$ model $\tilde{\alpha}_{k}$ :

$$
\tilde{\alpha}_{k}=a . \tilde{\alpha}_{k-1}+e_{k}
$$

where $e_{k}$ is a white circular complex Gaussian noise with variance $\sigma_{e}^{2}=\left(1-a^{2}\right) \sigma_{\alpha}^{2}$. The observation equation (5) is then approximated by:

$$
y_{k}=s_{k} \tilde{\alpha}_{k}+n_{k}
$$

The AR(1) coefficient verifies

$$
a=\frac{R_{\tilde{\alpha}}[1]}{R_{\tilde{\alpha}}[0]}
$$

In the literature, the correlation matching (CM) criterion is usually imposed [2], [4]. It means that the autocorrelation of the exact channel gain $\alpha$ and the AR(1) approached process $\tilde{\alpha}$ coincide for the first two coefficients (i.e., $R_{\tilde{\alpha}}[0]=R_{\alpha}[0]=$ $A^{2} \sigma_{\alpha_{1}}^{2} \sigma_{\alpha_{2}}^{2}$, and $R_{\tilde{\alpha}}[1]=R_{\alpha}[1]$ ), and using equation (16) and (13) the AR(1) coefficient $a$ noted $a_{C M}$ becomes

$$
a_{C M}=J_{0}\left(2 \pi f_{d 1} T\right) J_{0}\left(2 \pi f_{d 2} T\right)
$$

The figure 2 shows the autocorrelation functions for both dual Rayleigh link and AR(1)-CM cases, at two values of Doppler frequencies. When the channel is varying slowly, that means when we have low Doppler frequencies (right figure, with $f_{d 1} T=f_{d 2} T=10^{-3}$ ), we see that the $\operatorname{AR}(1)$ $\mathrm{CM}$ autocorellation function seems to stay constant, since the
$\mathrm{AR}(1)$ coefficient $a_{C M} \approx 1$ (see eq. (17), with $J_{0}(\varepsilon) \approx 1$ when $0 \leq \varepsilon<<1$ ). Then, the matching is less convincing in case of low frequencies, contrary to the situation with high frequencies where the autocorrelation function is less out of tune (left figure, with $f_{d 1} T=f_{d 2} T=10^{-1}$ ). Based on this observation, we will not consider the CM criterion to compute the AR(1) parameter in the present study but a variance criterion, as already proposed for the one-link channel in [10] and [11]. The choice of the AR(1) parameter $a$ will then be based on minimizing the asymptotic estimation error variance (MAV) as developed in the section III.A, and we will call it $a_{M A V}$.

\section{Kalman filter $(K F)$}

Given the model and observation equations (14) and (15), we use a KF to get an on-line unbiased estimate, $\hat{\alpha}_{k}$, of the true $\alpha_{k}$. The error is $\varepsilon_{k}=\alpha_{k}-\hat{\alpha}_{k}$, with variance $M S E=E\left\{\left|\varepsilon_{k}\right|^{2}\right\}$.

In this work, we concentrate of the performance of the channel estimator. So we treat a simplified case assuming the symbols are known (pilot-aided scenario) or perfectly decided (decision-directed scenario), thus we assume $s_{k}=1$. Note that in practice, our channel estimator can be easily coupled with a detector in order to perform joint channel estimation and decision tasks, for example via the Expectation-Maximization algorithm framework (see [13]).

The KF for this simplified case is given by (see p. 436 in [14]):

$\begin{array}{ll}\text { Prediction } & \hat{\alpha}_{k \mid k-1}=a \hat{\alpha}_{k-1} \\ \text { Prediction MSE } & P_{k \mid k-1}=a^{2} P_{k-1}+\sigma_{e}^{2} \\ \text { Kalman Gain } & K_{k}=\frac{P_{k \mid k-1}}{\sigma_{n}^{2}+P_{k \mid k-1}} \\ \text { Correction } & \hat{\alpha}_{k}=\hat{\alpha}_{k \mid k-1}+K_{k}\left(y_{k}-\hat{\alpha}_{k \mid k-1}\right) \\ \text { MSE } & P_{k}=\left(1-K_{k}\right) P_{k \mid k-1}\end{array}$

Replacing (ii) in (iii), (v) in (ii), and (i) in (iv), we obtain the simplified system:

$$
\begin{aligned}
K_{k} & =\left[a^{2} P_{k-1}+\sigma_{e}^{2}\right] /\left[a^{2} P_{k-1}+\sigma_{e}^{2}+\sigma_{n}^{2}\right] \\
P_{k} & =\left(1-K_{k}\right)\left(a^{2} P_{k-1}+\sigma_{e}^{2}\right) \\
\hat{\alpha}_{k} & =a \hat{\alpha}_{k-1}+K_{k}\left(y_{k}-a \hat{\alpha}_{k-1}\right)
\end{aligned}
$$

where $K_{k}$ is the Kalman gain at iteration $k$ and $P_{k}$ is the estimation error variance.

Without loss of generality, we assume $E_{1}=1$ and $\sigma_{\alpha_{1}}^{2}=\sigma_{\alpha_{2}}^{2}=$ 1. Given (6),

$$
\sigma_{\alpha}^{2}=A^{2}
$$

For the special case where $\sigma_{n_{1}}^{2}=\sigma_{n_{2}}^{2}$, given (7)

$$
\sigma_{n}^{2}=A^{2} \sigma_{n_{1}}^{2}+\sigma_{n_{2}}^{2}=\sigma_{n_{1}}^{2}\left(A^{2}+1\right)
$$

So for this case, and given (11), we have

$$
\gamma=\frac{A^{2}}{\sigma_{n_{1}}^{2}\left(A^{2}+1\right)}=\gamma_{1} \frac{A^{2}}{A^{2}+1}
$$

We clearly see that $\gamma<\gamma_{1}$. 

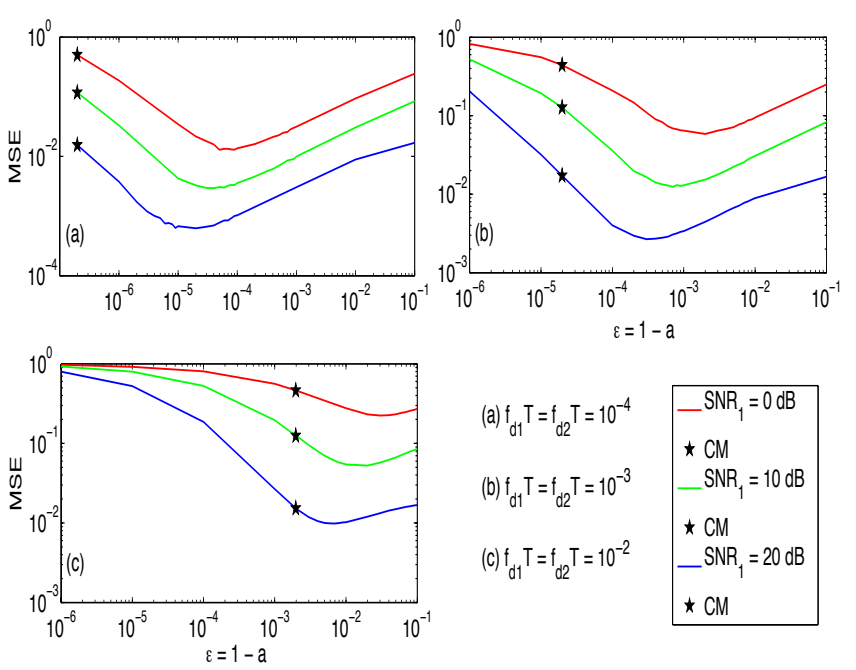

Figure 3. MSE in function of $\varepsilon=1-a$ for different Doppler frequencies $f_{d 1} T=f_{d 2} T=f_{d} T$. The $\star$ indicates the position of $M S E_{C M}$ and the corresponding $1-a_{C M}$.

\section{Simulation AND RESUlts}

We present Monte Carlo simulations to demonstrate the channel estimation error variance of AF relaying. We apply the Kalman filter for the CM case where $a=a_{C M}$, and calculate the corresponding error variance $M S E_{C M}$ as first step. As a second step, we apply the AR(1) Kalman filter for many other cases where $a$ is taken manually, and found the $M S E$ for each case. At the end, we choose the optimal value of $a$, denoted $a_{M A V}{ }^{1}$, that minimizes the $M S E$.

In our simulations, we are in the special case of $\sigma_{n_{1}}^{2}=\sigma_{n_{2}}^{2}$, and we take $A=1$. Then $\sigma_{n}^{2}=2 \sigma_{n_{1}}^{2}$ and $\gamma=\frac{\gamma_{1}}{2}$, similarly to say $S N R=S N R_{1}-3 d B$ where $S N R_{1}$ is the signal to noise ratio for the S-R link.

\section{A. Tuning of the AR(1)-parameter}

The figure 3 shows the error variance in function of $\varepsilon=$ $1-a$. We see that at low frequencies, the minimum value of $M S E$ is attended for $\varepsilon_{M A V}>>\varepsilon_{C M}$, i.e, for $a_{M A V}<a_{C M}$ while we can see that this difference is more reduced when the Doppler frequency and/or the $S N R$ increase. Comparing the values of the MSE in the "valley" (i.e. in the minimum region) and the one obtained with $\mathrm{CM}$ criterion, $M S E_{C M}$, we observe that the CM criterion is far from optimal, leading to a strong loss in performance in terms of $M S E$. The strict minimum is noted $M S E_{M A V}$, from which we can measure the related $\mathrm{AR}(1)$-parameter, $a_{M A V}$.

\footnotetext{
${ }^{1}$ Note that once the values of $a_{M A V}$ for different values of $S N R$ and Doppler frequencies are stored in tables, we can use them for the on-line estimation.
}

\begin{tabular}{|c|c|c|c|}
\hline$\frac{a_{C M}}{a_{M A V}}$ & $f_{d 1} T=1 e-4$ & $f_{d 1} T=1 e-3$ & $f_{d 1} T=1 e-2$ \\
\hline$f_{d 2} T=1 e-4$ & $\frac{0.9999998}{0.9999500}$ & $\frac{0.9999900}{0.9991000}$ & $\frac{0.9990131}{0.9800000}$ \\
\hline$f_{d 2} T=1 e-3$ & $\frac{0.9999900}{0.9991000}$ & $\frac{0.9999802}{0.9980000}$ & $\frac{0.9990034}{0.9800000}$ \\
\hline$f_{d 2} T=1 e-2$ & $\frac{0.9990131}{0.9800000}$ & $\frac{0.9990034}{0.9800000}$ & $\frac{0.9980275}{0.9700000}$ \\
\hline
\end{tabular}

Table I

COMPARISON BETWEEN $a_{C M}$ AND $a_{M A V}$ FOR DIFFERENT DOPPLER FREQUENCIES AT $S N R=0 \quad d B$

\begin{tabular}{|c|c|c|c|}
\hline$\frac{a_{C M}}{a_{M A V}}$ & $f_{d 1} T=1 e-4$ & $f_{d 1} T=1 e-3$ & $f_{d 1} T=1 e-2$ \\
\hline$f_{d 2} T=1 e-4$ & $\frac{0.9999998}{0.9999800}$ & $\frac{0.9999900}{0.9998000}$ & $\frac{0.9990131}{0.9960000}$ \\
\hline$f_{d 2} T=1 e-3$ & $\frac{0.9999900}{0.9998000}$ & $\frac{0.9999802}{0.9997000}$ & $\frac{0.9990034}{0.9950000}$ \\
\hline$f_{d 2} T=1 e-2$ & $\frac{0.9990131}{0.9960000}$ & $\frac{0.9990034}{0.9960000}$ & $\frac{0.9980275}{0.9930000}$ \\
\hline
\end{tabular}

Table II

COMPARISON BETWEEN $a_{C M}$ AND $a_{M A V}$ FOR DIFFERENT DOPPLER FREQUENCIES AT $S N R=20 d B$

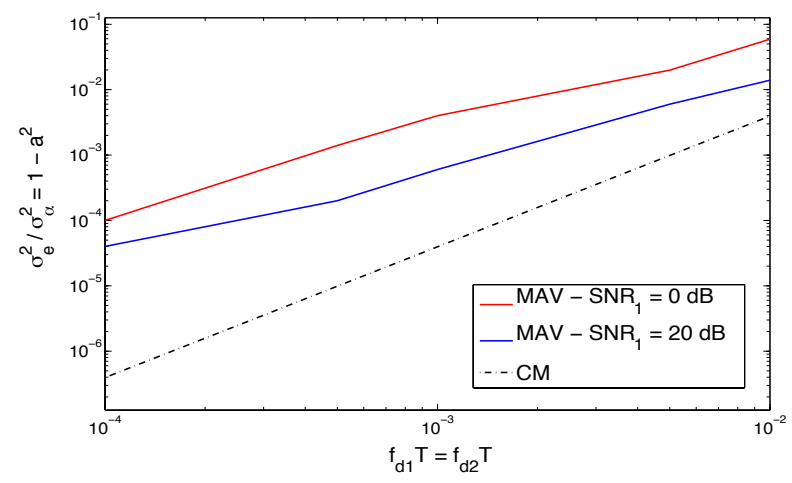

Figure 4. State noise variance $\sigma_{e}^{2} / \sigma_{\alpha}^{2}=1-a^{2}$ used in the AR(1)-model in function of Doppler frequency for MAV and CM cases

In tables I and II, we can compare the values of $a_{M A V}$ and $a_{C M}$. It is clear, especially for low Doppler frequencies, that the CM coefficient is higher than the optimal coefficient. Thus, when we are in the optimal case, the AR(1)-based autocorrelation curve decreases more rapidly to zero and approaches better the values of the Bessel-based autocorrelation function (13) than it was in figure 2 for the CM case.

In figure 4 , we have plotted the state noise variance $\sigma_{e}^{2}$ used in the Kalman filter as a function of $f_{d} T$. We recall that the state noise variance is related to the AR(1)-parameter $a$ by $\sigma_{e}^{2}=\left(1-a^{2}\right) \sigma_{\alpha}^{2}$. We can measure by this figure how much the state noise variance of the CM criterion is too weak compared to the optimal value (MAV criterion), leading about a factor 100 for $f_{d} T=10^{-4}$, and about a factor 10 for $f_{d} T=10^{-2}$.

It should be noted that the variation of $\sigma_{e}^{2}$ versus $f_{d} T$ in a $\log / \log$ scale is linear with a slope of 2 (i.e. $20 \mathrm{~dB} /$ decade). It can be explained analytically by an approximation of 


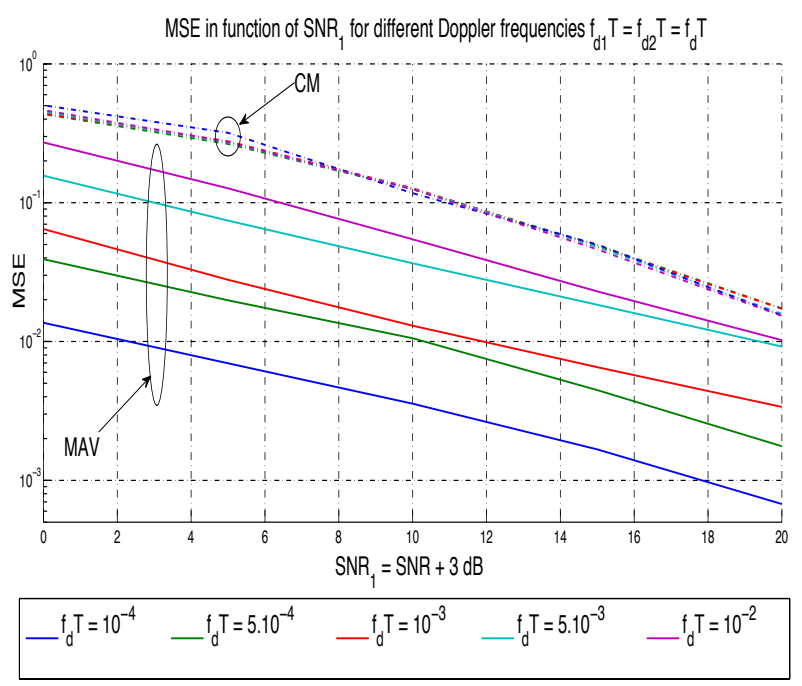

Figure 5. Comparison between $M S E_{C M}$ and $M S E_{M A V}$ in function of signalto-noise ratio for different Doppler frequencies

the Bessel function : $\left[J_{0}\left(2 \pi f_{d} T\right)\right]^{2} \approx 1-\left(2 \pi f_{d} T\right)^{2} / 2$ when $2 \pi f_{d} T<<1$, leading to $\frac{\sigma_{e}^{2} C M}{\sigma_{\alpha}^{2}}=1-a_{C M}^{2} \approx\left(2 \pi f_{d} T\right)^{2}$.

\section{B. Same Doppler frequencies}

In this case, we suppose the source and the destination moving at the same speed. We note $f_{d 1} T=f_{d 2} T=f_{d} T$.

In figure 5, we plot the error variance for Correlation Matching case $\left(M S E_{C M}\right)$ in function of $S N R$, and compare it to the minimum error variance we reached $\left(M S E_{M A V}\right)$. The plots are dressed for different Doppler frequencies $f_{d} T$.

We see that $M S E_{C M} d B$ and $M S E_{M A V} d B$ varies linearly with $S N R_{1} \mathrm{~dB}$. We deduce then that $M S E_{M A V}=K \cdot \gamma^{\beta}$. Graphically, we can calculate the coefficient $\beta$

$$
\beta=\frac{\Delta\left[\log _{10}\left(M S E_{M A V}\right)\right]}{\Delta\left[\log _{10}(\gamma)\right]}=10 \frac{\Delta\left[\log _{10}\left(M S E_{M A V}\right)\right]}{\Delta[S N R]}
$$

We obtain $\beta \approx-0.67 \approx-\frac{2}{3}$

In figure 6, we plot the error variance for Correlation Matching case $\left(M S E_{C M}\right)$ in function of $f_{d} T$, and compare it to the minimum error variance we reached $\left(M S E_{M A V}\right)$. The plots are dressed for different $S N R$. We see too that $M S E_{M A V} d B$ vary linearly with $\log _{10}\left(f_{d 1} T\right)=\log _{10}\left(f_{d 2} T\right)=\log _{10}\left(f_{d} T\right)$, while $M S E_{C M}$ seems constant with respect to $f_{d} T$. In the same way, we can say that $M S E_{M A V}=K^{\prime} \cdot\left(f_{d} T\right)^{\zeta}$ and we calculate graphically the coefficient $\zeta$. We obtain

$$
\zeta=\frac{\Delta\left[\log _{10}\left(M S E_{M A V}\right)\right]}{\Delta\left[\log _{10}\left(f_{d} T\right)\right]}
$$

We obtain $\zeta \approx 0.69 \approx \frac{2}{3}$.

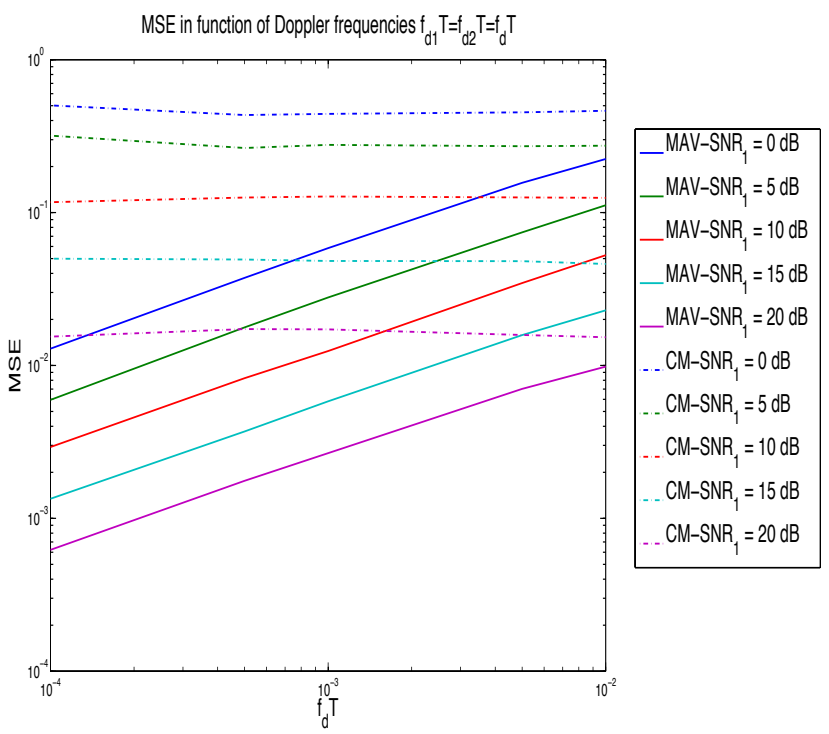

Figure 6. Comparison between $M S E_{C M}$ and $M S E_{M A V}$ in function of Doppler frequency for different signal-to-noise ratio

Finally, and given $\gamma=\frac{1}{\sigma_{n}^{2}}$ in our simulations, we can say that

$$
M S E_{M A V}=C \cdot\left(f_{d} T \times \sigma_{n}^{2}\right)^{\frac{2}{3}}
$$

where $C$ is a constant value.

It is noteworthy that this optimal MSE for the AR(1) Kalmanbased estimation of the mobile-fixed-mobile relay channel (with two Clarkes' models) seems to take the same form than the one obtained for the one-link Clarkes'model channel established in [11], eq. (25) (i.e. with the same (2/3) power of $f_{d} T \times \sigma_{n}^{2}$ ).

\section{Different Doppler frequencies}

In this case, we suppose the source and the destination moving at different speeds. The same reasoning persists. The minimum error variance $M S E_{M A V}$ increases with $f_{d 1} T$ and $f_{d 2} T$, and with $S N R$, as can be observed in figure 6 .

\section{BER performances}

Until now, all the performances are dressed in terms of MSE. Finally, we have a look at the consequences in terms of Bit Error Rate (BER) in figure 8. This figure refers to a binary PSK (BPSK) transmitted over an Amplify-and-Forward dual Rayleigh fading channel with $f_{d 1} T=f_{d 2} T=10^{-3}$. In order to ensure convergence of the iterative detection and decoding algorithm, 10 pilot symbols (known at the receiver) every 100 code symbols is placed in the transmitted codeword ${ }^{2}$. So we

\footnotetext{
${ }^{2}$ To use the KF equations in (18), (19) and (20), which are given for known symbols case, i.e. for $s_{k}=1$ in (15), and since we have $s_{k} \in\{-1 ;+1\}$, the KF equations are modified by substituting $y_{k}$ with $y_{k} \times \hat{s}_{k \mid k-1}^{*}$ where $\hat{s}_{k \mid k-1}=s_{k}$ if $s_{k}$ is known (pilot) or $\hat{s}_{k \mid k-1}=\operatorname{sgn}\left\{\Re\left(\hat{\alpha}_{k-1}^{*} \times y_{k}\right)\right\}$ if $s_{k}$ is unknown (data). In this case, $\hat{s}_{k \mid k-1}$ represents the a priori decision, and the final decision will be $\hat{s}_{k}=\operatorname{sgn}\left\{\Re\left(\hat{\alpha}_{k}^{*} \times y_{k}\right)\right\}$.
} 

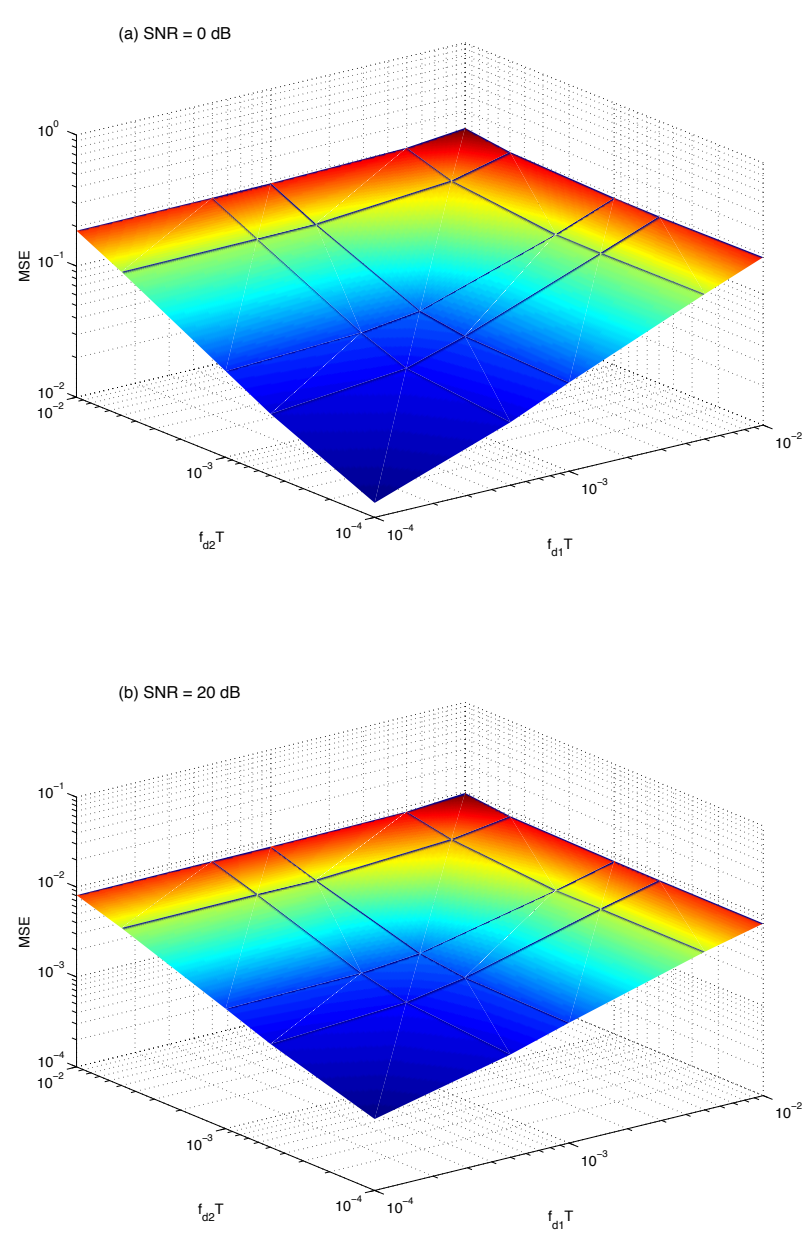

Figure 7. MSE $E_{M A V}$ in function of Doppler frequencies for high and low signal-to-noise ratios $S N R$

use a semi-blind pilot-assisted channel estimation. We show a significant improvement in BER performance after channel estimation with MAV-KF versus the CM-KF, especially in low SNR region.

\section{CONCLUSION}

This paper addresses the problem of estimating an Amplifyand-Forward channel using a first-order AR(1) model. Simulations show that the most widely used choice for the AR(1) pole estimation (the CM criterion) is not accurate for low SNR and low Doppler $f_{d} T$. Therefore, varying the value of the $\mathrm{AR}(1)$ coefficient, we carry out the optimization of the AR(1) model. It is demonstrated graphically that the $M S E_{M A V}$ of the $\mathrm{AR}(1) \mathrm{KF}$ is proportional to the $(2 / 3)$ power of the product $\left(f_{d} T \times \sigma_{n}^{2}\right)$, where $\sigma_{n}^{2}$ is the observation noise variance.

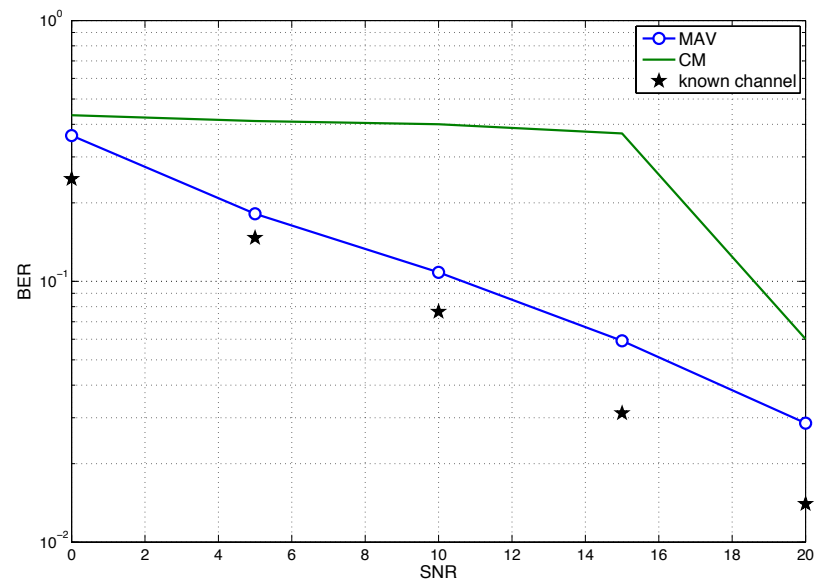

Figure 8. BER in function of SNR for $f_{d} T=10^{-3}$

\section{REFERENCES}

[1] C. S. Patel and G. L. Stüber , "Channel Estimation for Amplify and Forward Relay Based Cooperation Diversity Systems", in IEEE Trans. on Wireless Comm., vol. 6, no. 6, Jun. 2007.

[2] X. Zhou, T. A. Lamahewa and P. Sadeghi, "Kalman Filter-based Channel Estimation for Amplify and Forward Relay Communications", in 43th Asilomar Conference on Signals, Systems and Computers, Nov. 2009.

[3] F. Liu, Z. Chen, X. Zhang and D. Yang,"Channel Estimation for Amplify and Forward Relay in OFDM System", in Proc. IEEE 4th Wireless Communications, Networking and Mobile Computing Conference WiCOM, Oct. 2008.

[4] I. Nevat, G.W. Peters G.W., A. Doucet and J. Yuan, "Channel Tracking in Relay Systems via Particle MCMC", in IEEE Vehicular Technology Conference, San Francisco, USA, Sep. 2011.

[5] C. S. Patel, G. L. Stüber and T. G. Pratt, "Statistical Properties of Amplify and Forward Relay Fading Channels", in IEEE Trans. on Vehicular Tech., vol. 55, no. 1, Jan. 2006.

[6] A. Chakrabarti, A. de Baynast, A. Sabharwal, and B. Aazhang, "HalfDuplex Estimate-and-Forward Relaying: Bounds and Code Design", in IEEE International Symposium on Information Theory, Seattle, Washington, USA , Jul. 2006

[7] B. Akhbari, M. Mirmohseni, and M. R. Aref, "Compress-and-Forward Strategy for The Relay Channel With Non-Causal State Information", in IEEE International Symposium on Information Theory, Seoul, Korea, Jun. 2009

[8] K. E. Baddour and N. C. Beaulieu, "Autoregressive modeling for fading channel simulation", in IEEE Trans. Wireless Comm., vol. 4, no. 4, pp. 1650-1662, Jul. 2005.

[9] H. S. Wang and P. C. Chang, "On verifying the first-order Markovian Assumption for a Rayleigh Fading Channel Model", in IEEE Trans. on Vehicular Tech., vol. 45, no. 2, pp. 353-357, May 1996.

[10] A. Barbieri, A. Piemontese, G. Colavolpe, "On the ARMA Approximation for Frequency Channels Described by the Clarke Model with Applications to Kalman-based Receivers", in IEEE Trans. on Wireless Comm., vol. 8, no. 2, Feb. 2009.

[11] S. Ghandour-Haidar, L. Ros and J. M. Brossier,"On the use of first-order autoregressive modeling for Rayleigh Flat Fading Channel Estimation with Kalman filter", in ELSEVIER Signal Processing, vol. 92, pp. 601606, Feb. 2012

[12] A. S. Akki and F. Haber, "A Statistical Model of Mobile-to-Mobile Land Communication Channel", in IEEE Trans. on Vehicular Tech., vol. 35, no. 1 , Feb 1986.

[13] T. Y. Al-Naffouri, "An EM-based forward-backward Kalman for the estimation of time-variant channels in OFDM", in IEEE Trans. on Signal Proc., vol. 55, Jul. 2007

[14] Steven M. Kay, Fundamentals of Statistical Signal Processing - Estimation Theory. Prentice Hall, 1993. 